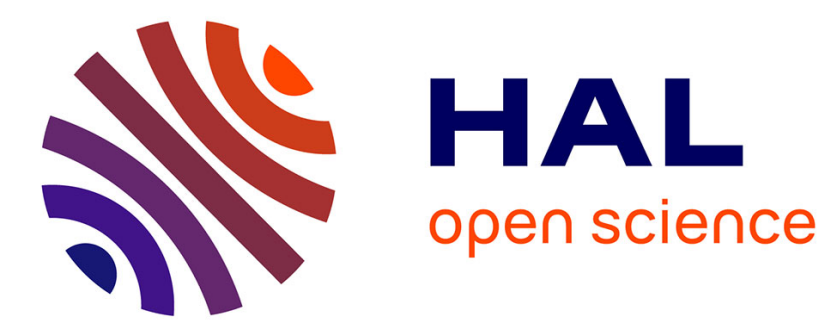

\title{
Système familial et relations d'attachement entre parents et enfants perçues par les frères et sœurs
}

Stéphanie Pinel-Jacquemin, Chantal Zaouche Gaudron

\section{To cite this version:}

Stéphanie Pinel-Jacquemin, Chantal Zaouche Gaudron. Système familial et relations d'attachement entre parents et enfants perçues par les frères et sœurs . Enfance, 2012, 2012 (02), pp.147 - 165. 10.4074/S0013754512002029 . hal-01498562

\section{HAL Id: hal-01498562 \\ https://hal-univ-tlse2.archives-ouvertes.fr/hal-01498562}

Submitted on 28 Apr 2017

HAL is a multi-disciplinary open access archive for the deposit and dissemination of scientific research documents, whether they are published or not. The documents may come from teaching and research institutions in France or abroad, or from public or private research centers.
L'archive ouverte pluridisciplinaire HAL, est destinée au dépôt et à la diffusion de documents scientifiques de niveau recherche, publiés ou non, émanant des établissements d'enseignement et de recherche français ou étrangers, des laboratoires publics ou privés. 
S. PINEL-JACQUEMIN*, Docteure, ATER

Université Toulouse ; UTM

Laboratoire Psychologie du Développement et Processus de Socialisation

Equipe «Milieux, Groupes et Psychologie du Jeune Enfant »,

5 allées Antonio Machado,

F-31058 TOULOUSE Cedex 9.

FRANCE

E-Mail : pinel-jacquemin@univ-tlse2.fr

C. ZAOUCHE GAUDRON. Professeure en Psychologie du Développement, Université Toulouse ; UTM

* : Auteur correspondant 


\section{ETAT DE LA QUESTION}

Lorsqu'à la fin des années soixante, John Bowlby développe sa théorie de l'attachement entre l'enfant et la personne la plus proche de lui, pourvoyeuse de soin principal, il focalise son attention en particulier sur la mère (1969). Celle-ci doit être disponible émotionnellement, sensible et capable de répondre aux signaux de détresse de son enfant pour que celui-ci soit sécurisé. Jusqu'à aujourd'hui, cette relation dyadique a suscité de nombreuses recherches en psychologie et, au-delà des caractéristiques des deux protagonistes, un certain nombre d'auteurs se sont attachés à démontrer que d'autres figures que la mère pouvaient servir de base de sécurité à l'enfant (Paquette, 2002 ; Pinel-Jacquemin, ZaoucheGaudron \& Troupel, 2009). Récemment Troupel et Zaouche-Gaudron (2004) ont également proposé que deux frères et sœurs puissent être attachés l'un à l'autre, c'est-à-dire -au sens de la théorie- rechercher auprès de l'autre la protection nécessaire lorsqu'ils se trouvent en situation de détresse. Ces auteures font l'hypothèse d'une base de sécurité «fraternelle » spécifique et complémentaire de celle élaborée avec les deux parents. Qu'il s'agisse de son père, de sa mère, d'un germain ou d'une autre personne, l'enfant, ayant trouvé le réconfort auprès de cette figure d'attachement, peut alors poursuivre l'exploration de son environnement. Il intègre ensuite progressivement ces expériences relationnelles en un Modèle Interne Opérant (M.I.O.) qui lui sert de prototype de relations sociales ultérieures (Bowlby, 1969).

Par ailleurs, des recherches ont également conduit à s'interroger sur la similarité ou la différence d'attachement mère-enfants perçus par les frères et sœurs. A l'aide de la procédure de la Situation Etrange ou de questionnaires d'évaluation de la qualité d'attachement parentenfant adressés à la mère, elles ont analysé la concordance d'attachement mère-enfants des frères et sœurs, c'est-à-dire vérifié si les deux germains avaient la même sécurité/insécurité d'attachement vis-à-vis de leur mère ou bien si on pouvait trouver un frère/sœur sécurisé et un 
autre insécurisé dans une même fratrie (Ricciuti, 1993; Sagi, Van Ijzendoorn, Aviezer, Donnell, Koren-Karie, Joels \& Harel, 1995 ; Ward, Vaughn \& Robb, 1988). Citons, par exemple, les résultats suivants : 62\% de concordance trouvés par Van Ijzendoorn, Moran, Belsky, Pederson, Bakermans-Kranenburg et Kneppers (2000) sur 138 paires de frères et sœurs de 3 à 5 ans (attachement mesuré par le protocole de la Situation étrange entre 12 et 14 mois pour chacun) ; $68 \%$ pour les enfants élevés en Kibboutz et donc non reliés biologiquement, dans l'étude de Sagi et et al., 1995, et enfin, 67\% de concordance trouvés par O’Connor et Croft (2001) dans leur étude sur 110 jumeaux de 42 à 45 mois. Ainsi, près d'un tiers des frères et sœurs n'ont pas le même attachement à leur mère. Les taux de concordance trouvés sont expliqués en premier lieu par des facteurs environnementaux plutôt que génétiques. Ces résultats conduisent les tenants de la «génétique comportementale » à aller au-delà des simples caractéristiques de la figure d'attachement (sensibilité, disponibilité et capacité à répondre aux signaux de détresse de l'enfant) et à s'intéresser à l'influence de l'environnement partagé, ou non partagé, des frères et sœurs pour comprendre les similitudes et les différences au sein de la fratrie (Plomin, 1994). Les facteurs environnementaux «non partagés » peuvent expliquer un fonctionnement différent des frères et sœurs, les facteurs «partagés », quant à eux, visent à rendre les frères et sœurs similaires. Parmi les facteurs «non partagés » les plus couramment étudiés, on trouve le traitement différentiel parental (différences de chaleur, de négativité, d'implication quotidienne, de contrôle parental, etc., reçus par les frères et sœurs), le tempérament et le sexe des enfants. Les facteurs «partagés » sont, par exemple, le statut socio-économique de la famille, le niveau d'étude de chacun des parents, etc.

L'impact de ces différentes composantes sur la qualité de l'attachement parent-enfant a fait alors l'objet de nombreuses recherches, sans, cependant, que ces composantes ne soient prises en compte simultanément. La théorie du système familial proposé par Minuchin (1974), 
selon laquelle une famille est un système composé d'éléments en interaction constituant des sous-systèmes à la fois autonomes et interdépendants (trangénérationnel, conjugal, coparental, parent-enfant et fraternel), interroge les influences entre ces différents sous-systèmes et les conséquences de celles-ci sur la perception de la qualité d'attachement de chacun des membres de la famille. La nécessité d'intégrer la théorie de l'attachement à celle de la théorie du système familial est d'ailleurs régulièrement relevée dans la littérature scientifique (ByngHall, 1995, 1999; Marvin \& Stewart, 1990). Nous avons donc recensé l'ensemble des composantes issues du système familial et ayant une influence sur la qualité de l'attachement parent-enfant (Pinel-Jacquemin \& Zaouche Gaudron, 2009 ; Pinel-Jacquemin, 2010). On peut les regrouper en trois catégories :

- celles qui concernent directement les parents, telles que leur représentation d'attachement (Steele \& al., 1996 ; Van Ijzendoorn, 1995), leur satisfaction conjugale (Davies \& Cummings, 1994 ; Erel \& Burman, 1995), leur alliance parentale (FivazDepeursinge \& Corboz-Warnery, 1999; McHale \& al., 2002), et leurs caractéristiques individuelles (Campbell \& al ., 2004 ; Harisson \& Ungerer, 2002),

- celles qui concernent directement les enfants et indirectement les parents par les représentations et attentes qu'elles induisent chez eux à l'égard de leur enfant : il s'agit du sexe (Tucker \& al., 2003 ; McHale \& al., 2000) et du tempérament des enfants (Balleyguier, 1991 ; Brody \& al., 1996 ; Crockenberg \& al., 2003 ; Dubeau \& Moss, 1998 ; Lovas, 2005),

- celles qui réunissent l'ensemble des représentations que se fait l'enfant des relations dans sa famille, telles que la perception qu'il a du style éducatif parental (Dekovic \& Janssens, 1992 ; Doyle \& al., 2004 ; Durning \& Fortin, 2000 ; Nair \& Murray, 2005 ; Tenenbaum \& Leaper, 1998), des conflits conjugaux (Erel \& al., 1998 ; Stocker \& al., 1997), du traitement différentiel parental (Crouter \& al., 1999 ; Feinberg \& al., 2000 ; McHale \& al., 2000 ; Tucker \& al., 2003 ; Volling \& Belsky, 1992), de ses relations fraternelles (Troupel \& 
Zaouche-Gaudron, 2004), et celles issues de son attachement à ses deux parents (Fox \& al., 1991 ; Goossens \& Van Ijzendoorn, 1990 ; Zaouche-Gaudron, 2002), et de l'attachement de deux frères et/ou sœurs à un même parent (Van Ijzendoorn \& al., 2000).

A partir de leur étude simultanée, conjointe, notre visée est de repérer celles qui fondent les différences individuelles de la qualité d'attachement entre deux enfants d'une famille (aîné, cadet) à une même figure d'attachement (père ou mère). Nous avons tenu compte de l'ensemble de ces variables dans notre modélisation et avons ajouté une variable «familiale globale» énoncée en termes de sécurité familiale afin de pouvoir situer celle-ci par rapport à la sécurité individuelle de chaque membre. L'objectif de cette étude est de vérifier notre hypothèse selon laquelle la perception des relations au sein de la famille influence la qualité de l'attachement parent-enfant. Cette perception est, selon nous, différente selon la place occupée par chacun au sein du système familial (père, mère, aîné, cadet, mais aussi parents, enfants, conjoints) et peut expliquer pourquoi deux frères et sœurs peuvent avoir une qualité d'attachement différente envers la même figure d'attachement.

\section{METHODE}

\subsection{Population}

Nos critères d'inclusion concernent les familles de nationalité française de deux enfants, âgés de six à douze ans, dont les quatre membres vivent sous le même toit. Le choix de la taille de la fratrie a été fait dans le but de limiter le nombre d'interactions (ou d' «alliances ») susceptibles d'influencer nos variables indépendantes (Camdessus, 1998). La période développementale se situe entre 6 et 12 ans, soit entre la petite enfance et l'adolescence. Avant 6 ans, les modèles d'attachement sont relativement labiles et perméables aux changements environnementaux (Bowlby, 1973). Quant à l'adolescence, il s'agit d'une période à la fois de forte ambivalence des sentiments et d'autonomisation plus grande par 
rapport aux parents, elle suppose donc d'être l'objet d'une étude à part entière. Les parents sont hétérosexuels, mariés ou non. La situation géographique est limitée à la métropole française, les départements ou territoires d'outre-mer et les pays francophones pouvant avoir des différences culturelles susceptibles d'influencer les pratiques familiales (Rothbaum \& Morelli, 2005).

Diverses sources de recueil des données ont été utilisées via un appel à participation pour accéder aux familles : il s'agit en premier lieu du bouche-à-oreille (46\%) : les collègues, les amis, les parents, les voisins et les connaissances diverses ont été sollicités. En second lieu, arrivent les forums en lien avec la famille ou la psychologie (32\%), puis les écoles primaires (20\%). 108 familles ont répondu à notre sollicitation. Elles sont issues de classes sociales favorisées. Les couples sont ensemble depuis 14.88 ans en moyenne $(E T=3.152)$. Les pères ont en moyenne 41 ans $(\mathrm{ET}=3.76)$, les mères 40 ans $(\mathrm{ET}=4.06)$, les aînés 10 ans $(\mathrm{ET}=1.56)$ et les cadets 7 ans et demi $(\mathrm{ET}=1.45)$. L'écart d'âge entre les deux enfants est en moyenne de 2.6 ans avec un écart-type de 1.06.

Les enfants peuvent faire partie d'une fratrie unisexe de filles ou de garçons (35\% des dyades « fille - fille » et $22 \%$ des dyades « garçon - garçon ») ou d'une fratrie mixte (19\% des dyades aînée fille-cadet garçon - ou 24\% des dyades aîné garçon-cadette fille).

\subsection{Instruments/variables ${ }^{1}$}

L'ensemble des variables étudiées a été évalué à partir d'auto-questionnaires anglosaxons validés au plan scientifique, traduits en français (validation française en cours). Le tableau suivant récapitule pour chacune d'elle le questionnaire utilisé. En ce qui concerne la mesure de «la qualité d'attachement parent-enfant» (CPAS ou Child-Parent Attachment Scale de Davies, Harold, Goeke-Morey \& Cummings, 2002), les enfants sont interrogés sur

\footnotetext{
${ }^{1}$ Tous les outils et informations les concernant sont disponibles auprès de l'auteur
} 
leurs perceptions d'attachement envers leur père et leur mère, les parents le sont sur leurs perceptions d'attachement à leurs enfants (aîné et cadet) et sur celles de leurs conjoints envers chaque enfant (exemple d'items pour cette dernière perception : "Quand mon enfant est contrarié, il va chercher du réconfort auprès de moi/mon/ma conjointe?»- Echelle de réponse en 5 points de $1=$ pas $d u$ tout à $5=$ tout-à-fait . La version enfants, composé de 15 items, a une consistance interne de $\alpha=.89$ et une fidélité tes-retest après deux semaines de $\mathrm{r}(92)=.83$; la version adressée aux parents comporte 18 items et a une consistance interne de $\alpha=.89$ également.

Une précision est également nécessaire en ce qui concerne la mesure de «l'alliance coparentale ». On sait que celle-ci représente le degré de soutien, ou au contraire de dénigrement, des parents entre eux dans leurs relations éducatives avec chacun de leurs enfants. Dans les modèles étudiés, le sigle ALLMA représente l'alliance coparentale perçue par le père dans sa relation avec la mère par rapport à l'aîné (par exemple, le père se sent soutenu par la mère lorsqu'il communique avec son aîné), le sigle ALLPC est celle qui est perçue par la mère dans sa relation avec le père par rapport au cadet, etc.

Concernant l'évaluation du «style éducatif parental perçu par les enfants », elle se base sur la définition de Baumrind $(1971$; 1989) qui fait apparaitre, sur deux axes de soutien et de contrôle, un style autoritaire (pas de soutien mais du contrôle), démocratique (soutien et contrôle), permissif (soutien, pas de contrôle) et désengagé ou détaché (pas de soutien, pas de contrôle).

Enfin, concernant la variable «sécurité familiale », il s'agit de la confiance qu'a chacun des membres dans sa famille en tant que soutien, protection et stabilité. Les mesures renseignent sur le sentiment de sécurité, de préoccupation ou de désengagement qu'a le répondant par rapport à son contexte familial global.

\section{Insérer tableau 1}




\subsection{Procédure}

Chaque membre de chaque famille a reçu un carnet de questionnaires portant sur les relations transgénérationnelles (père, mère), conjugales, coparentales, parents-enfants et fraternelles. La consigne a été donnée de répondre individuellement aux questionnaires et de ne pas influencer les réponses (en particulier du cadet). Le temps de réponses moyen aux questionnaires est estimé pour chaque membre à trois-quarts d'heure - une heure. Les résultats n'ont pu être pris en compte que si l'ensemble des questionnaires (père, mère, aîné, cadet d'une même famille) était retourné. Au total, ce sont $38 \%$ des questionnaires envoyés aux familles volontaires pour participer à l'étude qui nous ont été renvoyés.

\section{$2.4 \underline{\text { Analyse des données }}$}

Afin d'examiner l'influence simultanée de l'ensemble des composantes du système familial, nous avons procédé en plusieurs temps :

1. Nous avons, tout d'abord, testé la validité factorielle de nos construits. Si la contribution factorielle de certains items n'était pas significative, ces items étaient écartés. Pour chacun des questionnaires utilisés, nous avons procédé à une démarche en deux étapes (Anderson \& Gerbing, 1988): 1) une analyse factorielle confirmatoire pour vérifier que chaque facteur de la structure caractérise bien la variable théorique observée et 2) une analyse de la cohérence interne de la variable théorique afin de vérifier que les items qui la décrivent mesurent bien la même variable. Pour cette dernière nous avons choisi de calculer l'alpha de Cronbach (1951). Cet indice traduit un degré d'homogénéité d'autant plus élevé que sa valeur est proche de 1 et jugé comme acceptable à partir de .65 .

Pour ce faire, nous avons utilisé le logiciel AMOS (version 6) et la procédure d'estimation de maximum de vraisemblance (ML Maximum of Likelihood) qui utilise une 
solution itérative pour résoudre l'ensemble des équations impliquées dans le modèle. Afin de pouvoir comparer les scores obtenus par les différents membres d'une même famille sur une même variable, nous avons homogénéisé les échelles et gardé les mêmes items pour les membres impliqués. Cela explique que certains items soient moins corrélés que d'autres pour certaines échelles ${ }^{2}$.

2. Dans un second temps, une analyse de corrélation nous a permis de cibler les variables en lien avec chacune des perspectives d'attachement (parents, enfants, conjoints), puis nous avons procédé à une analyse de régression pour les variables présentant une corrélation significative (mais inférieure à 0.70 afin d'éviter tout risque de multicolinéarité) ${ }^{3}$.

3. Enfin, nous avons testé dans un modèle en pistes causales les variables dont la régression était significative. L'apport de ces méthodes par rapport aux démarches plus classiques telles que la régression linéaire ou multiple est de permettre, d'une part, le traitement d'estimations simultanées de plusieurs relations de dépendances inter-reliées, et d'autre part, l'incorporation d'erreurs de mesure directement dans le processus d'estimation (Roussel, Durrieu, Campoy \& Qkremi, 2002). La prise en compte des erreurs de mesure permet d'obtenir des informations plus précises que les méthodes classiques concernant les coefficients de régression (Hair, Anderson, Tatham, \& Black, 1998). Par ailleurs, les effets des déterminants sur les variables dépendantes peuvent être traités simultanément et permettent ainsi de reproduire une situation réelle et complexe, où chaque déterminant n'agit a priori jamais seul, mais de façon concomitante avec d'autres facteurs. Ce type de modèle permet également d'étudier les effets direct, indirect et total entre plusieurs variables et de tester les effets réciproques, ce qui permet alors de « hiérarchiser la force de la relation d'une variable sur l'autre » (Roussel \& al., 2002, 8). Ainsi les variables dépendantes peuvent, dans

\footnotetext{
${ }^{2}$ Analyses factorielles confirmatoires pour chaque variable disponibles auprès de l'auteur

${ }^{3}$ Analyses de corrélation et de régression pour chaque variable disponibles auprès de l'auteur
} 
un premier chemin causal, jouer un rôle différent que celui qu'elles jouent dans un second, où elles rempliront la fonction de variables indépendantes ou de variables médiatrices.

Les indices d'ajustement des modèles retenus sont, en plus du chi deux normé ou $\mathrm{X}^{2} / \mathrm{DDL}$, chi deux par degré de liberté $(C M I N / D F)$, deux indices absolus, la Root Mean Square Error of Approximation ou RMSEA (Steiger \& Lind, 1980) et l'Adjusted Goodnessof-Fit Index ou AGFI (Jöreskog et Sörbom, 1984), auxquels s'ajoutent deux indices incrémentaux de type 2 en ce qui concerne le Tucker-Lewis Index ou TLI (Tucker \& Lewis, 1973) et de type 3 pour le Comparative Fit Index ou CFI (Bentler, 1989 ; 1990). Le RMSEA représente la différence moyenne, par degré de liberté, qui est attendue dans la population totale et non dans l'échantillon. Il est indépendant de la taille de l'échantillon et de la complexité du modèle, contrairement au chi deux normé. Son intervalle de confiance est de 90\%. Il doit être inférieur à 0.08 et si possible à 0.05 (Roussel \& al., 2002).

L'AGFI mesure la part relative de la variance-covariance expliquée par le modèle ajusté par le nombre de variables par rapport au nombre de degrés de liberté. Analogue au $\mathrm{r}^{2}$ ajusté de la régression multiple, il est peu sensible à la taille de l'échantillon. En revanche il est sensible à la complexité du modèle. Nous le donnons à titre indicatif. Son seuil est généralement de 0.9 .

Le TLI compare le manque d'ajustement du modèle à tester à celui du modèle de base. Sa valeur permet d'estimer l'amélioration relative, par degré de liberté, du modèle à tester par rapport au modèle de base. Son seuil clé est également à 0.9 .

Le CFI mesure la diminution relative au manque d'ajustement. Celle-ci est estimée suivant la distribution non centrée du $\mathrm{X}^{2}$ du modèle à tester par rapport au modèle de base. Il est performant avec les petits échantillons et son seuil est fixé à 0.9 .

Enfin le dernier indice d'ajustement retenu est le CMIN/DF ou $\mathrm{X}^{2}$ par degré de liberté. Il doit être inférieur à 3. 
Le modèle de base auquel nous comparons nos données empiriques est le modèle nul (default model), c'est-à-dire un modèle pour lequel aucune relation structurelle entre les variables n'est prévue : autrement dit, un modèle dont les variables observées ne seraient pas corrélées. Ce modèle nul est le modèle restrictif comparatif le plus communément utilisé. Pour créer les modèles de données empiriques, nous avons utilisé, pour chaque perspective interrogée (parents, enfants, conjoints), les liens établis dans la littérature scientifique et conserver ceux dont les régressions confirmaient cette influence. Si le modèle présentait des indices d'ajustement insuffisants, nous procédions à une re-spécification posthoc du modèle à partir des indices de modifications fournis par le programme, tout en tenant compte, bien sûr, de l'apport théorique sous-jacent à cette modélisation.

\section{RESULTATS/DISCUSSION}

Voici le modèle testé, suite aux trois étapes décrites précédemment, pour les parents :

\section{Insérer Figure 1}

Ce modèle présente un très bon ajustement. Ses indices sont les suivants :

$X^{2}=92.060 ; \mathrm{DDL}=57$

\begin{tabular}{|c|c|c|c|c|c|c|}
\hline CMIN/DF & AGFI & TLI & CFI & RMSEA & LO90 & HI90 \\
\hline 1.615 & 0.806 & 0.936 & 0.965 & 0.076 & 0.046 & 0.103 \\
\hline
\end{tabular}

Voici maintenant celui qui concerne les perceptions d'attachement des enfants :

$$
\text { Insérer Figure } 2
$$

Les indices d'ajustement de ce modèle en pistes causales, rapportés ci-après, sont très bons : $X^{2}=59.241$, ddl $=46$ et :

\begin{tabular}{|c|c|c|c|c|c|c|}
\hline CMIN/DF & AGFI & TLI & CFI & RMSEA & LO90 & HI90 \\
\hline 1.288 & 0.845 & 0.954 & 0.973 & 0.052 & 0.000 & 0.087 \\
\hline
\end{tabular}


Enfin, celui des conjoints :

\section{Insérer Figure 3}

Les indices d'ajustement de ce modèle, rapportés ci-après, sont également très bons.

$\mathrm{X}^{2}=119.649 ; \mathrm{DDL}=87$

\begin{tabular}{|c|c|c|c|c|c|c|}
\hline CMIN/DF & AGFI & TLI & CFI & RMSEA & LO90 & HI90 \\
\hline 1.375 & 0.819 & 0.941 & 0.957 & 0.059 & 0.029 & 0.084 \\
\hline
\end{tabular}

Avant d'aller plus loin, notons que si ces modèles, au départ identiques, font finalement intervenir des variables différentes, c'est bien parce que certaines d'entre elles ne sont pas significatives dans les perceptions de tous les membres d'une même famille. A ce titre, elles ont été ôtées des modèles présentés, sauf lorsque leur absence avait une incidence négative sur les indices d'ajustement ${ }^{4}$.

Par rapport à la problématique posée, à savoir comprendre pourquoi deux enfants d'une même fratrie peuvent avoir un attachement de qualité différente avec une même figure d'attachement, ces résultats apportent un éclairage tout à fait innovant. En effet, lorsqu'on tient compte simultanément de l'ensemble des représentations des relations familiales, il apparaît clairement que les représentations familiales qui ont une influence sur la qualité de l'attachement sont très différentes d'un membre à l'autre et ce, quelle que soit la perspective étudiée (père-aîné, père-cadet, mère-aîné, mère-cadet, aîné-père, aîné-mère, cadet-père, cadetmère, père/mère-aîné, père/mère-cadet, mère/père-aîné, mère/père-cadet). Des différences dans les pistes causales peuvent ainsi être mises en avant :

\subsection{Pour le père (figure 1)}

\footnotetext{
${ }^{4}$ Les résultats des indices de corrélation pour chaque relation impliquée dans ces modèles sont à disposition auprès de l'auteur
} 
Les perceptions d'attachement du père sont très proches pour ses deux enfants (â̂né ou cadet). On remarque l'influence de la qualité de l'attachement à l'autre enfant et, dans le cas du cadet, également de la qualité de l'attachement mère-aîné. On voit ici l'importance pour le père d'une relation mère-aîné sécurisante pour qu'il se sente à son tour sécurisé à la fois avec l’aîné mais aussi avec le cadet.

Dans les deux cas (attachements père-aîné et père-cadet), le père est, par ailleurs, sensible à des variables des sous-systèmes conjugaux et coparentaux. La dépendance entre les sous-systèmes conjugaux et parentaux, mise en avant par Corwyn et Bradley (1999), Lamour (2000) ou encore Owen et Cox (1997), est ici aussi plus importante pour le père que pour la mère. Cette intrication est expliquée par Brody, Arias et Fincham (1996) selon la théorie du rôle social qui soutient que les femmes sont susceptibles de compartimenter leurs rôles de conjointes et de mères davantage que ne le font les hommes.

L'ensemble des relations entre les membres de la famille exerce, par ailleurs, une influence sur la qualité de l'attachement du père à ses enfants, ce qui souligne, de manière générale, la sensibilité importante de ce dernier au contexte émotionnel et affectif familial (Schermerhorn \& al., 2008).

\subsection{Pour la mère (figure 1)}

Les perceptions qu'a la mère de ses attachements à l'aîné et au cadet sont très différentes l'une de l'autre. L'attachement mère-cadet, contrairement à l'attachement mèreaîné, est indépendant du reste de la famille : ici, les composantes conjugales et coparentales sont absentes. Tout se passe comme si la mère prenait appui sur le père dans la mise en place de son attachement à l'aîné, mais qu'elle pouvait s'en détacher pour le cadet. On peut également faire l'hypothèse d'une parentalité évolutive, dans le sens où ce recul peut s'avérer 
plus difficile pour la mère avec un premier enfant, alors qu'il deviendra plus aisé avec l'élargissement de la famille.

Par ailleurs, contrairement à ce qu'avaient suggéré les auteurs cherchant à comprendre la forte concordance d'attachement d'un enfant à ses deux parents aucune caractéristique des enfants (sexes, tempéraments), dans notre étude, n'entre en jeu dans la perception maternelle de l'attachement parent-enfant (Rosen \& Rothbaum, 1993 ; Seifer \& Vaughn, 1995).

\subsection{Pour les enfants (figure 2)}

Certaines variables jouent un rôle pour l'aîné et aucun pour le cadet : c'est le cas du style éducatif paternel qui influence la perception d'attachement de l'aîné à son père et n'entre pas en considération pour le cadet. Dans notre population, une majorité des aînés le décrit comme «permissif» $(74 \%)$, c'est-à-dire soutenant mais pas contrôlant. Nous pouvons expliquer cette perception par le statut socio-économique élevé des familles, qui d'après Bellerose et al. (2002) et Durning et Fortin (2000), va de paire avec un meilleur soutien affectif.

Dans notre modèle, le style éducatif de la mère est fortement corrélé à celui du père, il intervient aussi dans la perception de l'aîné, mais médié par la sécurité familiale de celui-ci. Ces résultats rejoignent, précisent et complètent ceux de Nair et Murray (2005) et de Karavasilis et al. (2003), qui trouvent une contribution directe du style éducatif maternel sur l'attachement mère-enfant. Ces auteurs suggèrent qu'un parentage qui promeut l'individualité de l'enfant et lui procure à la fois du soutien et des réponses à ses attentes, favorise l'établissement d'une représentation de soi comme digne d'être aimé et de sa mère comme étant disponible, c'est-à-dire d'un Modèle Interne Opérant (M.I.O.) sécurisé. Le manque d'études concernant le lien entre le style éducatif du père et cette perception de l'attachement par l'enfant ne nous permet pas de discuter ce résultat au regard d'autres travaux. En attendant 
que d'autres recherches viennent le confirmer, nous pouvons faire l'hypothèse que l'explication est la même que celle apportée pour la mère et qu'un parentage paternel soutenant et cadrant favorise la perception de sécurité de l'enfant dans sa relation au père, ce que disent d'ailleurs Kromelow, Harding et Touris (1990).

On peut remarquer, par ailleurs, que les deux enfants sont sensibles à la qualité de l'attachement de leur germain dans leur propre perception d'attachement à leur mère : le cadet est sensible à la sécurité d'attachement de l'aîné à sa mère, mais pour l'aîné c'est la sécurité familiale du cadet qui importe pour qu'il se sente sécurisé dans sa relation à sa mère. D'une manière générale, on peut dire que la sécurité familiale de tous les membres de la famille est importante dans la perception de sécurité de l'aîné à chacun de ses parents. C'est le cas aussi pour la perception d'attachement du cadet à sa mère, mais pas à son père, où seules interviennent les sécurités familiales du cadet et du père eux-mêmes.

Notre modèle en pistes causales précise que l'attachement à la mère perçu par l'aîné prédit l'attachement au père (et non l'inverse), alors que pour le cadet, ce lien est réciproque. Nous pensons pouvoir rapporter cette différence au fait que dans les familles favorisées, l'implication du père est plus importante (Darling-Fisher \& Tiedje, 1990) et que le père prend davantage confiance dans ses capacités paternelles avec un second enfant et devient ainsi plus sensible aux signaux de détresse du cadet qu'il ne l'était pour l'aîné.

Enfin, il est intéressant de relever (cf. figure 2) le lien entre l'attachement de l'aîné à sa mère et celui du cadet à sa mère, le premier prédisant le second et qui confirme, tout en lui donnant un sens (de l'aîné vers le cadet), la concordance entre l'attachement de deux enfants à leur mère trouvée, entre autres, par Van Ijzendoorn et al. (2000).

\subsection{Pour les conjoints (figure 3)}


On notera ici l'influence de l'alliance parentale pour le père : plus il se sentira soutenu par la mère dans son rôle éducatif envers chacun des enfants - mais plus particulièrement pour l'aîné -, plus il percevra leur attachement à leur mère comme sécurisé. Les caractéristiques des enfants (âge, sexe, tempérament) ne jouent aucun rôle dans sa perception.

Pour la mère, ce sont davantage ses propres caractéristiques (dépression, temps de travail) et celles de l'aîné (âge et sexe) qui vont jouer un rôle dans sa perception de l'attachement des enfants à son conjoint. Plus l'aîné est âgé, plus la mère perçoit son attachement au père comme insécurisé. Il se peut ici que l'autonomie de l'aîné évolue de façon parallèle à une moindre implication du père. Cette hypothèse est soutenue par la proportion de style éducatif « détaché » trouvée dans la dyade père-aîné, bien plus importante que dans toutes les autres. Le fait que l'aînée soit une fille est également pris en compte par la mère dans sa perception (aussi bien pour l'aîné que pour le cadet). Ce résultat rejoint ceux de Schoppe-Sullivan et al. (2006) pour qui le sexe de l'enfant est clairement associé à la sécurité de l'attachement père-enfant et non mère-enfant, mais contredit ceux de Dubeau et Moss (1998) et Lovas (2005) pour qui les dyades mères-filles ont des attachements plus sécurisés que les dyades pères-filles.

Ainsi les sous-systèmes impliqués sont très différents selon qu'on adopte la perception d'un des membres de la famille ou un autre. Nous les récapitulons dans le tableau ci-après (les cases colorées indiquent une influence du sous-système) :

\section{Insérer tableau 2}

\section{CONCLUSION}

Au travers du prisme plus large que nous proposons d'utiliser pour saisir ce qui fonde la qualité de l'attachement parent-enfant, nous avons montré que les représentations familiales 
influencent différemment les membres d'une même famille, selon leurs rôles et leurs places, dans leurs perceptions d'attachement. Ces différences permettent de comprendre pourquoi deux enfants d'une même famille peuvent avoir un attachement de qualité différente à une même figure d'attachement, et ce, quelle que soit la perspective interrogée (enfants, parents, conjoints).

Les principaux résultats issus de cette recherche indiquent, qu'exceptée la perception d'attachement de la mère pour son cadet, les parents sont davantage sensibles à des variables conjugales (satisfaction conjugale) et coparentales (alliances coparentales) qu'à des variables parentales ou personnelles; la qualité d'attachement mère-aîné semble influencer celle des autres dyades.

Pour les enfants, la perception de leurs attachements tient compte de l'attachement des germains à leur mère. L'aîné est davantage sensible aux sécurités familiales de l'ensemble des membres de la famille que le cadet. Enfin le style éducatif paternel joue un rôle dans la perception d'attachement de l'aîné à son père et aucune incidence pour le cadet.

En ce qui concerne les perceptions des conjoints, ce sont l'alliance parentale et les caractéristiques individuelles des enfants (âge, sexe) et de la mère (dépression, temps de travail) qui entrent en compte. Le père apparaît comme également moins autonome dans sa perception d'attachement de sa conjointe, ce qui pourrait confirmer sa difficulté à séparer les sphères conjugale, coparentale et parentale.

Malgré la complexité des modèles invoqués, les résultats obtenus dans cette étude apportent un éclairage nouveau sur la compréhension de l'attachement parent-enfant et élargit les perspectives de recherche dans le domaine. 


\section{Références bibliographiques}

Anderson, J. C., \& Gerbing, D. W. (1988). Structural equation modeling in practice: a review and recommended two-step approach. Psychological Bulletin, 103, 411-423.

Balleyguier, G. (1991). Le développement de l'attachement selon le tempérament du nouveau-né. Psychiatrie de l'Enfant, 34(2), 641-657.

Bellerose, C., Cadieux, E., \& Noël, Y. (2002). Milieu familial des jeunes Québécois de 9 ans, 13 ans et 16 ans. In ISQ, Enquête sociale et de santé des enfants et des adolescents québécois 1999. Québec : Institut de la Statistique du Québec.

Bentler, P. M. (1989). EQS Structural Equations Program Manual. Los Angeles: BMDP Statistical Software.

Bentler, P. M. (1990). Comparative fit indexes in structural models. Psychological Bulletin, $107,238-246$.

Bowlby, J. (1969). Attachement et perte. L'Attachement, vol. 1. Paris : PUF (1978, pour la première édition française).

Bowlby, J. (1973). Attachement et perte. La séparation : angoisse et colère, vol. 2. Paris : PUF (1978, pour la première édition française).

Brody, G. H., Arias, I., \& Fincham, F. (1996). Linking marital and child attributions to family processes and parent-child relationships. Journal of Family Psychology, 10, 408421.

Byng-Hall, J. (1995). Creating a secure family base: some implications of attachment theory for family therapy. Family Process, 34, 45-58.

Byng-Hall, J. (1999). Family and couple therapy. In J. Cassidy \& P. Shaver (Eds.), Handbook of Attachment: Theory, Research and Clinical Implications (pp. 625-645). New York: Guilford Press.

Camdessus, B. (1998). La fratrie méconnue, liens du sang, lien du coeur. Paris : ESF.

Corwyn, R. F., \& Bradley, R. H. (1999). Determinants of paternal and maternal investment in children. Infant Mental Health Journal, 20, 238-256.

Crockenberg, S. (1986). Are temperamental differences in babies associated with predictable differences in care-giving? In J. Lerner \& R. Lerner (Eds.), Temperament and psychosocial interaction in children: New directions for child development, 31 (pp. 53-73). San Francisco: Jossey-Bass.

Crockenberg, S., \& Leerkes, E. (2003). Infant negative emotionality, caregiving, and family relationships. In A. C. Crouter \& A. Booth (Eds.), Children's influence on family dynamics: The neglected side of family relationships (pp. 57-78). Mahwah, NJ, US: Lawrence Erlbaum Associates, Publishers.

Crouter, A. C., McHale, S. M., \& Tucker, C. J. (1999). Does stress exacerbate parental differential treatment of siblings? A pattern-analytic approach. Journal of Family Psychology, 13(2), 286-299.

Darling-Fisher, C. S. \& Tiedje, L. B. (1990). The impact of maternal employment characteristics on fathers participation in child care. Family Relations, 39, 20-26.

Dekovic, M., \& Janssens, J. M. A. M.(1992). Parents'child-rearing style and child's sociometric status. Developmental Psychology, 28, 925-932. 
Doyle, B. A., Moretti, M. M., Brendgen, M., \& Bukowski, W. (2004). Parent-child relationships and adjustment in adolescence : Findings from the HBSC Cycle 3 and NLSCY Cycle 2 studies. Retrieved December 12, 2005, from http://www.phac-aspc.gc./ca/dcadea/publications/pcr-rpe/index.html

Dubeau, D., \& Moss, E. (1998). La théorie de l'attachement résiste-t-elle au charme des pères? Approche comparative des caractéristiques maternelles et paternelles durant la période d'âge préscolaire de l'enfant. Enfance, 3, 82-102.

Durning, P., \& Fortin, A. (2000). Les pratiques éducatives parentales vues par les enfants. Enfance, 4, 375-391.

Erel, O., Margolin, G., \& John, R. S. (1998). Observed sibling interaction: Links with the marital and the mother-child relationship. Developmental Psychology, 34(2), 288-298.

Feinberg, M. E., Neiderhiser, J. M., Simmens, S., Reiss, D., \& Hetherington, E. M. (2000). Sibling comparison of differential parental treatment in adolescence: Gender, self-esteem, and emotionality as mediators of the parenting-adjustment associations. Child Development, 71, 1611-1628.

Fox, N. A., Kimmerly, N. L., \& Schafer, W. D. (1991). Attachment to Mother/Attachment to Father: A Meta-Analysis. Child Development, 62, 210-225.

Goossens, F. A., \& Van Ijzendoorn, M. H., (1990). Quality of infants' attachments to professional caregivers: Relation to infant-parent attachment and day-care characteristics. Child Development, 61, 832-837.

Hair, J. F., Anderson, R. E., Tatham, R. L., \& Black, W. C. (1998). Multivariate data analysis with readings, 5th edition. Upper Saddle River, NJ: Prentice-Hall.

Jöreskog, K., \& Sorbom, D. (1984). Lisrel IV: Analysis of linear structural relationships by maximum likelihood, instrumental variables and least square methods ( ${ }^{\text {rd }}$ Ed.). Mooresville, IN: Scientific Software.

Karavasilis, L., Doyle, A. B., \& Markiewicz, D. (2003). Associations between parenting style and attachment to mother in middle childhood and adolescence. International Journal of Behavioral Development, 27(2), 153-165.

Komelow, S., Harding, C., \& Touris, M. (1990). The role of the father in the development of stranger sociability during the second year, American Journal of Orthopsychiatry, 60, 521530.

Lamour, M. (2000). Paternalité et interactions familiales père-mère-nourrisson. Thèse de Doctorat d'Etat. Paris : Université René Descartes.

Lovas, G. S. (2005). Gender and patterns of Emotional Availability in Mother-toddler and Father-toddler Dyads. Infant Mental Health Journal, 26(4), 327-353.

Nair, H., \& Murray, A. D. (2005). Predictors of attachment security in preschool children from intact and divorced families. The Journal of Genetic Psychology, 166(3), 245-263.

O'Connor, T. G., \& Croft, C. M. (2001). A twin study of attachment in preschool children. Child Development, 72(5), 1501-1511.

Owen, M. T., \& Cox, M. J. (1997). Marital conflict and the development of infant-parent attachment relationships. Journal of Family Psychology, 11, 152-164.

Marvin, R. S., \& Stewart, R. B. (1990). A family system framework for the study of attachment. In M. T. Greenberg, D. Chiccetti \& E. M. Cummings (Eds), Attachment in the 
preschool years: Theory, research and Intervention (pp. 51-86), Chicago: University of Chicago Press.

McHale, S. M., Updegraff, K. A., Jackson-Newsom, J., Tucker, C. J., \& Crouter, A. C. (2000). When does Parental Differential Treatment have negative implications for siblings? Blackwell Publishers Ltd.

Minuchin, S. (1974). Families and Family Therapy. Harvard University Press, Cambridge. Trad. Fr. (1998) : Familles en Thérapie. Ramonville Saint Agne : Edition Erès.

Nair, H., \& Murray, A. D. (2005). Predictors of attachment security in preschool children from intact and divorced families. The Journal of Genetic Psychology, 166(3), 245-263.

Paquette, D. (2002). Du nouveau sur la relation d'attachement père-enfant. Défi jeunesse, 8(2), 6-12.

Pinel-Jacquemin, S. (2010). L'attachement parent-enfant dans une perspective systémique. In C. Zaouche Gaudron (Ed.), Le Développement social de l'enfant. Paris : Dunod, Collection Les Topos (3è édition).

Pinel-Jacquemin, S., \& Zaouche-Gaudron, C. (2009). Système familial et attachement parentenfant : revue de la question. Neuropsychiatrie de l'Enfant et de l'Adolescent, 57(3), 167172.

Pinel-Jacquemin, S., Zaouche-Gaudron, C., \& Troupel, O. (2009). Attachement Père-Enfant et Qualité des Relations Fraternelles Cadet-Aîné. Psychologie Française, 54, 307-322.

Plomin, R. (1994). Nature, nurture, and social development. Social Development, 3(1), 37-53.

Ricciuti, A. E. (1993). Child-mother attachment: A twin study. Poster presented at the Sixtieth Anniversary Meeting of the Society for research in Child Development, March 25-28, New Orleans.

Rosen, K. S., \& Rothbaum, F. (1993). Quality of parental caregiving and security of attachment. Developmental Psychology, 29(2), 358-367.

Rothbaum, F., \& Morelli, G. (2005). Attachment and culture: Bridging relativism and universalism. In W. Friedlmeier, P. Chakkarath \& B. Schwarz (Eds), Culture and human development: The importance of cross-cultural research for the social sciences (pp. 99123). Hove, UK: Psychology Press.

Roussel, P., Durrieu, F., Campoy, E., \& Qkremi, A. (2002). Méthodes d'équations structurelles : recherche et applications en gestion. Paris : Economica.

Sagi, A., Van Ijzendoorn, M. H, van, Aviezer, O., Donnell, F., Koren-Karie, N., Joels, T., \& Harel, Y. (1995). Attachment in a multiple caregiver and multiple infant environment: The case of the Israeli kibbutzim. In E. Waters, B. E. Vaughn, G. Posada, \& K. KondoIkemura (Eds.), Constructs, cultures and caregiving: New growing points of attachment theory. Monographs of the Society for Research in Child Development, 60 (2-3, Serial $N^{\circ}$ 244), (pp. 71-91).

Schermerhorn, A. C., Cummings, E. M., \& Davies, P. (2008). Children's representations of multiple family relationships: Organizational structure and development in early childhood. Journal of Family Psychology, 22(1), 89-101.

Schoppe-Sullivan, S. J., Diener, M. L., Mangelsdorf, S. C., Brown, G., L., McHale, J. L., \& Frosch, C. A. (2006). Attachment and sensitivity in family context: the role of parent and infant gender. Infant and Child Development, 15, 367-385. 
Seifer, R., \& Vaughn, B. (1995). Mastery motivation within a general organizational model of competence. In R. H. MacTurk, G. A. Morgan, \& E. J. Hrncir (Eds.), Mastery motivation: Origins, conceptualizations, and applications: Vol. 12. Advances in applied developmental psychology (pp. 95-115). Norwood, NJ: Ablex.

Steiger, J. H., \& Lind, J. C. (1980, Mai). Statistically based tests for the number of factors. Paper presented at the annual spring meeting of the Psychometric Society, Iowa City, IA.

Stocker, C., Ahmed, K., \& Stall, M. (1997). Marital satisfaction and maternal emotion expressiveness: links with children's sibling relationships. Social Development, 6(3), 373 385.

Tenenbaum, H. R., \& Leaper, C. (1998). Gender effects on Mexican-descent parents' questions and scaffolding during toy play: A sequential analysis. First Language, 18(53), 129-147.

Troupel, O., \& Zaouche-Gaudron, C. (2004). Le lien d'attachement fraternel : l'utilisation des histoires à compléter. Revue Internationale de l'Education Familiale, 8(1), 27-38.

Tucker, L. R., \& Lewis, C. (1973). The reliability coefficient for maximum likelihood factor analysis. Psychometrika, 38, 1-10.

Tucker, C. J., McHale, S. M., \& Crouter, A. C. (2003). Dimensions of mother's and father's Differential Treatment of siblings: Links with adolescent's sex-typed qualities. Family Relationships, 52, 82-89.

Van Ijzendoorn, M. H., Moran, G., Belsky, J., Pederson, D., Bakermans-Kranenburg, M. J., \& Kneppers, K. (2000). The similiraty of sibling's attachment to their mother. Child Development, 71(4), 1086-1098.

Volling, B. L., \& Belsky, J. (1992). The Contribution of Mother-Child and Father-Child Relationships to the Quality of Sibling Interaction: A longitudinal study. Child Development, 63, 1209-1222.

Ward, M. J., Vaughn, B. E., \& Robb, M. D. (1988). Social-emotional adaptation and infantmother attachment in siblings: role of the mother in cross-sibling consistency. Child Development, 59(3), 643-651.

Zaouche-Gaudron, C. (2002). Le développement social de l'enfant: du bébé à l'âge scolaire. Paris : Dunod. 
TABLEAUX \& FIGURES

Tableau 1 : Récapitulatif des variables mesurées et des outils correspondants

\begin{tabular}{|c|c|c|}
\hline SOUS-SYSTEMES & VARIABLES & OUTILS \\
\hline Transgénérationnel & $\begin{array}{c}\text { Représentation } \\
\text { d'attachement des parents }\end{array}$ & $\begin{array}{c}\text { P-AASQ } \\
\text { Parent-Adult Attachment Style } \\
\text { Questionnaire } \\
\text { (Behrens \& Lopez, 1998) }\end{array}$ \\
\hline \multirow[t]{2}{*}{ Conjugal } & Satisfaction conjugale & $\begin{array}{l}\text { Echelle d'ajustement dyadique } \\
\text { (Spanier, 1976) }\end{array}$ \\
\hline & $\begin{array}{l}\text { Représentation par les } \\
\text { enfants des conflits } \\
\text { conjugaux }\end{array}$ & $\begin{array}{c}\text { Children Perception of Interparental } \\
\text { Conflict Scale (CPIC) + Young-CPIC } \\
\text { (Grych, 2000) }\end{array}$ \\
\hline Coparental & $\begin{array}{c}\text { Alliance parentale des } \\
\text { parents }\end{array}$ & $\begin{array}{c}\text { Parenting Alliance Inventory PAI } \\
\text { (Abidin \& Brunner, 1994) }\end{array}$ \\
\hline \multirow[t]{3}{*}{ Parent-enfant } & Style éducatif parental & $\begin{array}{c}\text { Pratiques Educatives Parentales Perçues } \\
\text { par les Enfants PEPPE } \\
\text { (Fortin, Cyr \& Chénier, 1997) }\end{array}$ \\
\hline & $\begin{array}{l}\text { Attachements Parents- } \\
\text { Enfants } \\
\text { (Perspectives des parents, des } \\
\text { enfants et des conjoints) }\end{array}$ & $\begin{array}{c}\text { Child-Parent Attachment Security CPAS- } \\
\text { Children \& Parents } \\
\text { (Davies \& al., 2002) }\end{array}$ \\
\hline & $\begin{array}{c}\text { Représentation du } \\
\text { Tempérament des Enfants }\end{array}$ & $\begin{array}{c}\text { Emotionality, Activity, Sociability EAS- } \\
\text { Parents } \\
\text { (Gasman \& al., 2002) }\end{array}$ \\
\hline \multirow[t]{2}{*}{ Fraternel } & $\begin{array}{l}\text { Représentation du } \\
\text { Traitement Différentiel } \\
\text { Parental }\end{array}$ & $\begin{array}{l}\text { SIDE Sibling Inventory Differential } \\
\text { Experiences - Traitement Parental } \\
\text { (Daniels \& Plomin, 1985) }\end{array}$ \\
\hline & Interaction fraternelle & $\begin{array}{l}\text { SIDE Sibling Inventory Differential } \\
\text { Experiences - Interaction fraternelle } \\
\text { (Daniels \& Plomin, 1985) }\end{array}$ \\
\hline FAMILLE & Famille $=$ Base de sécurité & $\begin{array}{l}\text { SIFS Security in the Family System } \\
\text { (Forman \& Davies, 2005) }\end{array}$ \\
\hline
\end{tabular}


Tableau 2 : Sous-systèmes intervenant dans la perception d'attachement parent-enfant selon les trois perspectives : parents, enfants et conjoints

\begin{tabular}{|c|c|c|c|c|c|c|c|c|c|c|c|c|c|}
\hline & \multicolumn{12}{|c|}{ PERSPECTIVES D'ATTACHEMENT PARENTS-ENFANTS } \\
\hline & & \multicolumn{4}{|c|}{ PARENTS } & & & \multicolumn{2}{|c|}{ ENFANTS } & \multicolumn{4}{|c|}{ CONJOINTS } \\
\hline & & \multicolumn{2}{|c|}{ PERE } & \multicolumn{2}{|c|}{ MERE } & \multicolumn{2}{|c|}{ AINE } & \multicolumn{2}{|c|}{ CADET } & \multirow{2}{*}{\multicolumn{2}{|c|}{$\begin{array}{c}\text { PERE } \\
\text { MERE-QINE MERE- CQDEET }\end{array}$}} & \multirow{2}{*}{\multicolumn{2}{|c|}{\begin{tabular}{|c|} 
MERE \\
PERE-gINE|PERE-CQ. DET
\end{tabular}}} \\
\hline & & AINE & CADET & AINE & CADET & PERE & MERE & PERE & MERE & & & & \\
\hline s & $\begin{array}{l}\text { Transgéné- } \\
\text { rationnel }\end{array}$ & & & & & & & & & & & & \\
\hline $\begin{array}{l}\text { iv } \\
3\end{array}$ & Conjugal & & & & & & & & & & & & \\
\hline s & Coparental & $M-A / M-C$ & MAA & $\begin{array}{c}\text { M-C/ } \\
\text { P.A/P-C }\end{array}$ & & $M-C \mathbb{P} \cdot C$ & & $M-C / P \cdot C$ & $M-C / P-C$ & & $M-C$ & & s-C \\
\hline $\begin{array}{l}1 \\
\vdots \\
T \\
\end{array}$ & Parental & $\begin{array}{l}\text { Attach } \\
\text { P.C }\end{array}$ & $\begin{array}{l}\text { Attach } \\
\text { P.A/M-A }\end{array}$ & $\begin{array}{c}\text { Attach } \\
\text { C M }\end{array}$ & $\begin{array}{l}\text { Attach } \\
\text { M-A }\end{array}$ & $\begin{array}{l}\text { Attach A.M } \\
\text { Sted A.P }\end{array}$ & & $\begin{array}{l}\text { Attach } \\
C \cdot M\end{array}$ & $\begin{array}{c}\text { Atach } \\
\text { C.P/A.M } \\
\end{array}$ & $\begin{array}{l}\text { Attach } \\
\text { PMC }\end{array}$ & Attach PMA & $\begin{array}{l}\text { Attach } \\
\text { MPC }\end{array}$ & Attach MPC \\
\hline \multirow{2}{*}{$\begin{array}{c}\mathrm{E} \\
\mathrm{M} \\
\mathrm{E} \\
\mathrm{S}\end{array}$} & $\begin{array}{l}\text { Caractérist. } \\
\text { parentales. }\end{array}$ & & & & & & Dépressio & maternelle & & & & Temps de & travail Mère \\
\hline & $\begin{array}{l}\text { Caractérist. } \\
\text { enfants } \\
\end{array}$ & & & & & & & & & & & \begin{tabular}{|l|} 
Sexe \& \\
Agge aîné \\
\end{tabular} & Sexe aîné \\
\hline E & Père & & & & & & $\begin{array}{l}\text { via Sécu } \\
\text { Fam } A\end{array}$ & & & & & & \\
\hline $\begin{array}{l}c \\
\text { u } \\
R\end{array}$ & Mère & & & & & $\begin{array}{l}\text { via Sécu } \\
\text { Fam A }\end{array}$ & $\begin{array}{l}\text { via Sécu } \\
\text { Fan A } A\end{array}$ & & $\begin{array}{c}\text { via Sécu } \\
\text { Fam } A \& \text { A Att } \\
\text { A-M }\end{array}$ & & & & \\
\hline $\begin{array}{c}F \\
A\end{array}$ & Aîné & & & & & & & & & & & & \\
\hline & Cadet & & & & & $\begin{array}{l}\text { via Sécu } \\
\text { Famp } P\end{array}$ & $\begin{array}{l}\text { via Sécu } \\
\text { Fân } A\end{array}$ & $\begin{array}{l}\text { via Sécu } \\
\text { Fañ } P\end{array}$ & & & & & \\
\hline
\end{tabular}

M : Mère, P : Père, A : Aîné, C : Cadet ; Sécu Fam : sécurité familiale, Att : Attachement 


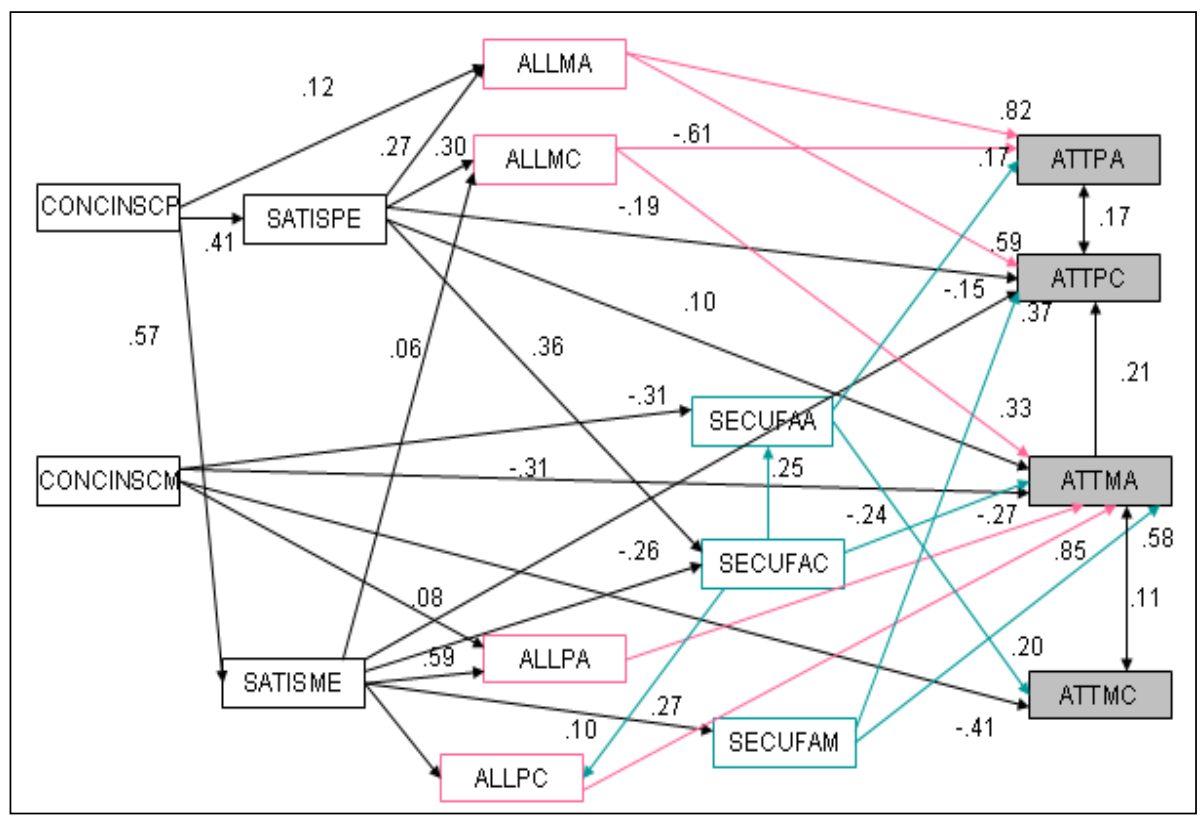

CONCINSP : concordance d'insécurité du Père

\section{ATTPA : Attachement Père-Aîné}

CONCINSM : concordance d'insécurité de la mère

ATTPC : Attachement Père-Cadet

SATISPE : satisfaction conjugale du père

ATTMA : Attachement Mère-Aîné

SATISME : satisfaction conjugale de la mère

ATTMC : Attachement Mère-Cadet

ALLMA : Alliance coparentale reçue de la mère et perçue par le père dans sa relation à son aîné
ALLMC :

Alliance coparentale perçue par le père dans sa relation à son cadet

ALLPA : Alliance coparentale perçue par la mère dans sa relation à son aîné

ALLPC : Alliance coparentale reçue du père et perçue par la mère dans sa relation à son cadet SECUFAA : Sécurité Familiale Aîné

SECUFAC : Sécurité Familiale Cadet SECUFAM : Sécurité Familiale Mère

Par souci de simplification, les estimations des erreurs et leurs corrélations ont été enlevées du schéma.

Figure 1 : Chemin en pistes causales de l'attachement parents-enfants, tels que perçus par les parents 


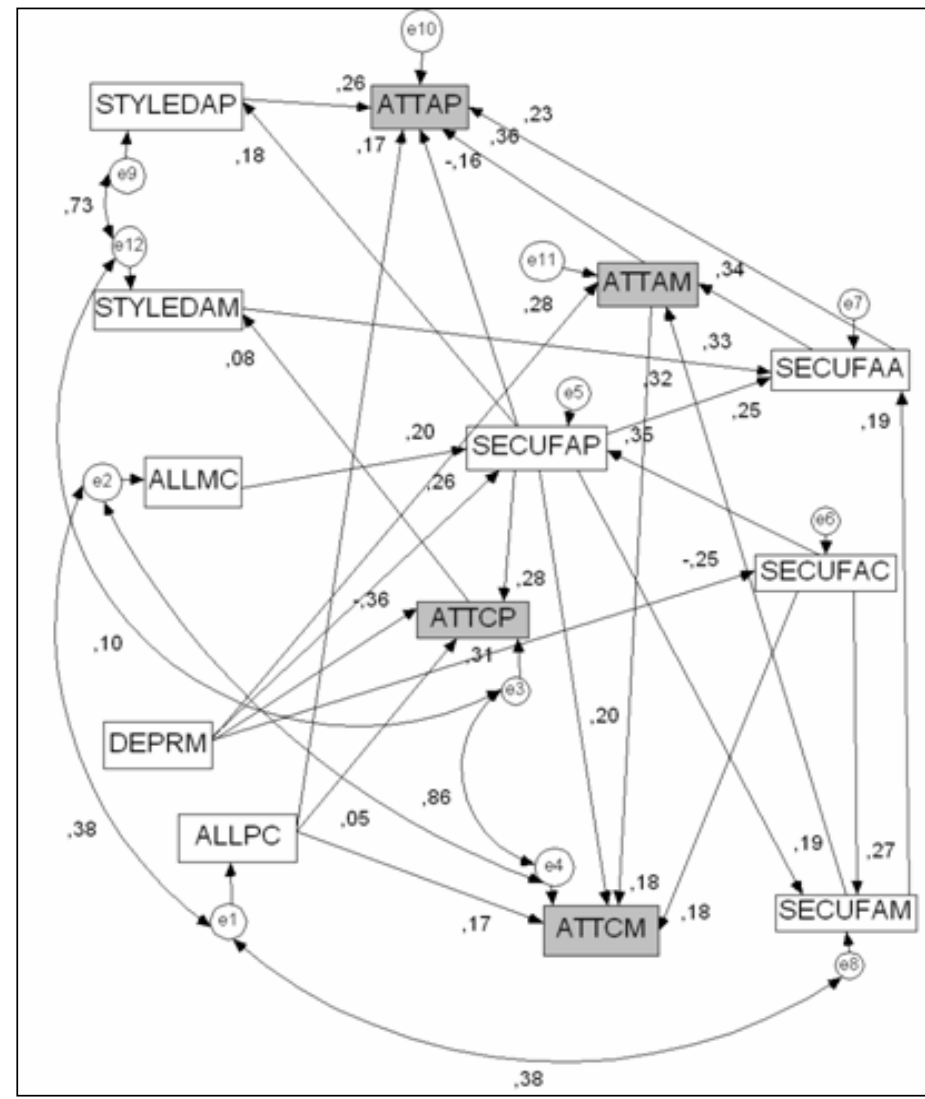

DEPRM : Dépression maternelle

ALLMC : Alliance coparentale Mère-Cadet (perception du père)

ALLPC : Alliance coparentale Père-Cadet (perception de la mère)

SECUFAM : Sécurité Familiale de la Mère ATTAP : Attachement Aîné-Père
ATTAM : Attachement Aîné-Mère ATTCP : Attachement Cadet-Père ATTCM : Attachement Cadet-Mère STYLEDAP : Style éducatif Père perçu par l'aîné STYLEDAM : Style éducatif Mère perçu par l'aîné SECUFAP : Sécurité Familiale du Père SECUFAA : Sécurité Familiale de l'Aîné

Figure 2 : Modèle en pistes causales des attachements parents-enfants tels que perçus par les enfants 


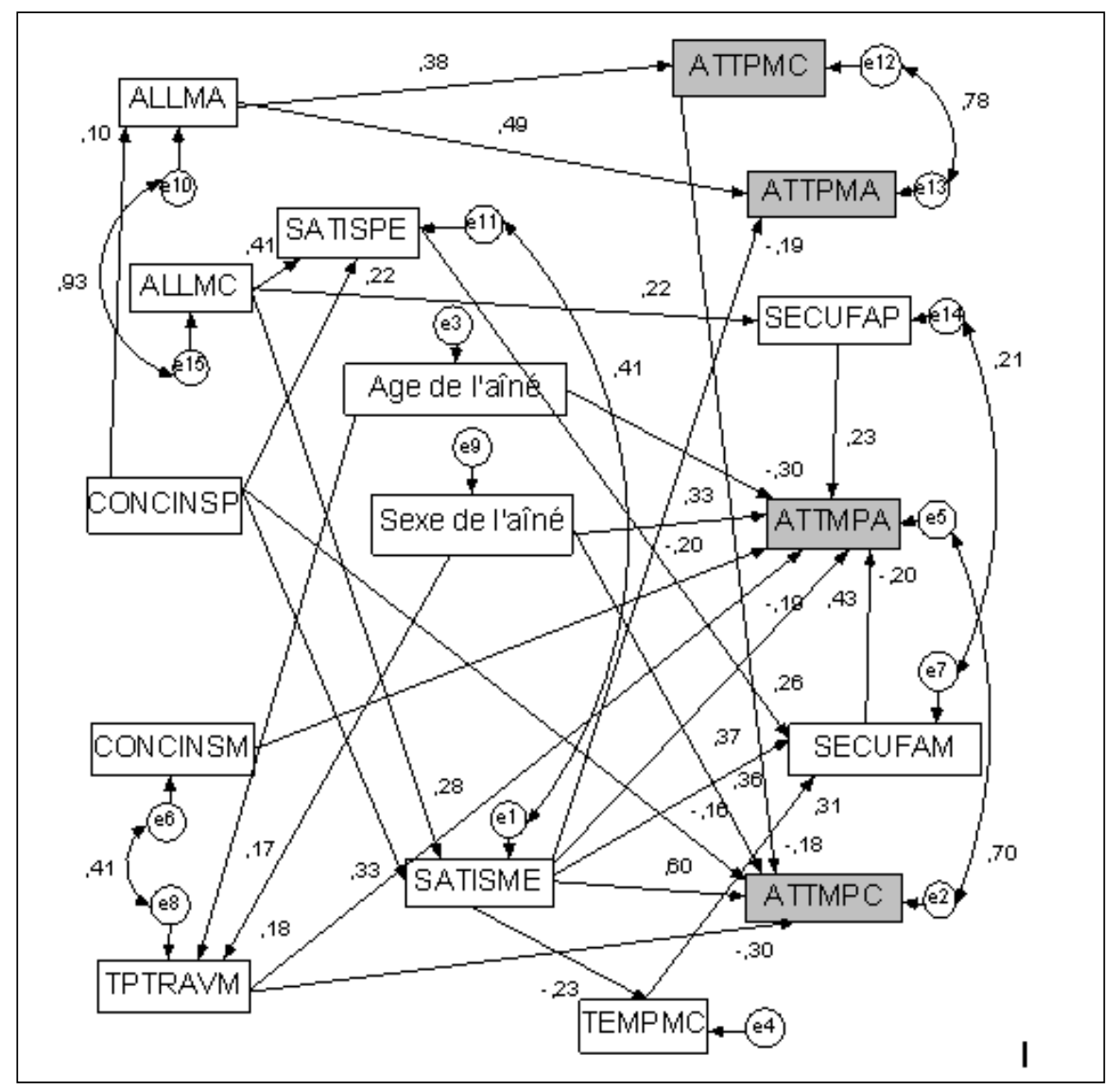

CONCINSP : Concordance d'attachements insécurisés du père CONCINSM : Concordance d'attachements insécurisés de la mère TPTRAVM : Temps de travail de la mère

TEMPMC : Décalage de tempérament du cadet perçu par la mère ALLMA : Alliance coparentale Mère-Aîné (perception du père) ALLMC : Alliance coparentale Mère-Cadet (perception du père) SATISPE : Satisfaction conjugale du Père

SATISME : Satisfaction conjugale de la Mère
ATTPMA : Attachement Mère-Aîné perçu par le Père ATTPMC : Attachement Mère-Cadet perçu par le Père

ATTMPA : Attachement Père-Aîné perçu par la Mère ATTMPC : Attachement Père-Cadet perçu par la Mère

SECUFAM : Sécurité Familiale Mère SECUFAP : Sécurité Familiale Père, Sexe de l'aîné ( $1=$ garçon, $2=$ fille)

Figure 3 : Modèle en pistes causales des attachements parents-enfants tels que perçus par les conjoints 